\title{
Improvement of Speed Measuring Precision for Traffic Radar
}

\author{
Xinjie $\mathrm{Wu}$ \\ College of engineering and technology \\ Beijing Institute of Economics and Management \\ Beijing,China \\ e-mail: wuxinjie@biem.edu.cn
}

\begin{abstract}
Aiming at improving the speed precision especially when there are more than one targets run through radar speed instrument at one time, we discuss the relation between the key performance parameters and speed precision as well as speed resolution. Then the main defects of the current traffic speed radars are analyzed and improvement measures are provided. Finally we provide a solution for multi-target circumstance. The solution uses STFT to improve time-frequency resolution. The simulation results show that the speed error decrease to less than when adopting the algorithm proposed in the paper.
\end{abstract}

Keywords-Doppler speed radar; Speed precision; speed resolution; Frequency resolution; Time-Frequency Analysis

\section{INTRODUCTION}

Traffic radar apparatus has been developed in order to secure vehicle travel safety [1]. But there are still many problems in practice though traffic speed radar has good application foreground. Especially when there are more than one target run through radar speed instrument at one time, it is more difficult to satisfy precision requirement [2]. So it is very important to do research on the theory and algorithm.

Aiming at improving the speed precision and speed resolution, we advise to choose appropriate frequency and adopt acceleration compensation to improve speed measuring precision. If multiple vehicles enter the stream at the same time, it becomes the officer's challenge to determine which vehicle is registering a particular speed with the radar device. So we propose a method based on timefrequency analysis to identify multi objects. The method can be used to simulating Doppler radar echo signals separation and parameter estimation and enhance speed resolution.

\section{IMPROVEMENT OF SINGLE TARGET SPEED PRECISION}

\section{A. Choosing of frequency}

Police microwave radars all use the Doppler Principle to measure speed. The radar transmits a continuous microwave signal and simultaneously measures the echo that is frequency shifted (Doppler shift) proportional to speed [3]. Obviously, the higher is the work frequency, the more is the number of Doppler pulses recorded in measuring period. So the speed resolution in high-frequency-region is higher than that of low-frequency-region. Therefore by choosing a higher work frequency, the measuring time can be compressed and the speed precision can be improved.

\section{B. Compensation of acceleration}

When microwave energy is reflected by a moving target, there is a shift in frequency. All Doppler radars utilize this principle. The amount of frequency shift is directly proportional to the target's velocity relative to the radar's transmitter. However, vehicles always run in uneven-velocity which leads to the decrease of speed decision.

Figure 1 show the frequency spectrum of even-velocity target and that of even-velocity target respectively.

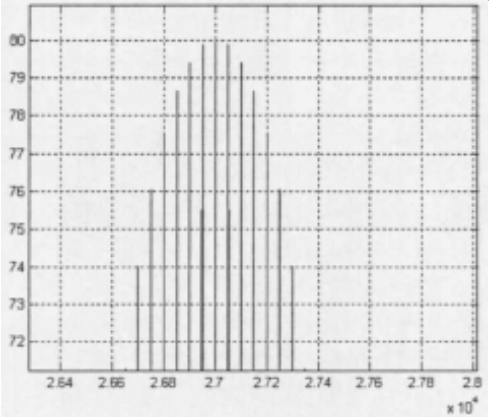

(a) even-velocity target

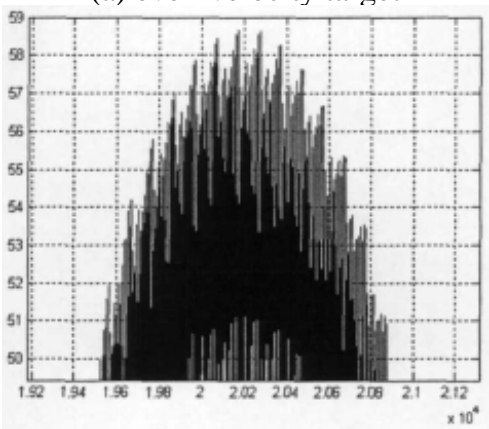

(b) uneven-velocity target

Figure 1. the frequency spectrum of moving target

From figure 1, we can find that the spectrum line will diffuse when the target has a bigger acceleration. As a result, the closed loop track can not be implemented for the appendix Doppler frequency shift led by the acceleration. The Doppler frequency shift led by the acceleration can be calculated utilizing the following formula [4].

$$
\frac{d \varphi_{a}(\tau)}{d \tau}=\frac{2 \pi}{\lambda} \cdot 2 a \tau
$$

So compensation of acceleration is utilized. Firstly, use multi-phase transformation to obtain target acceleration 
value. Then, implement accelerate compensation using the value. Finally analyze the compensated signal to obtain target speed value.

\section{MUlTI TARGETS CIRCUMSTANCE}

\section{A. Multi targets solution}

Microwaves (radar) signals obey the Doppler Principle which is a frequency shift that results from relative motion between a frequency source and a listener. The Doppler shift is directly proportional to speed between source and listener, frequency of the source, and the speed the wave travels (speed of light for microwaves). But traditional methods in traffic monitoring radar have the problem that it can not distinguish the overspeed target from several parallel vehicles [5].

The identification of several vehicles is a difficult problem in radar speed field. There are three factors which are relative to echo power: the distance between target and radar, the beam width of radar antenna, the angle between target and beam centre, the size and material of reflective plane. These three factors determine the amplitude of echo power. So the measuring methods based on amplitude are used for multiple targets circumstance.

Take two-lane targets measuring as example. When each lane has vehicle passing, two radar apparatus which are installed over two lanes can receive both echo signals. The difference between their positions leads to the difference of Doppler component and energy of received echo signals. The measuring diagram is shown in Figure 2. Firstly judge whether there is overspeed vehicle. If there is, judge how many overspeed vehicles there are. If there is only one overspeed vehicle, judge the position of overspeed vehicle by analyzing the maximum doppler frequency component. If there are two overspeed vehicles, record the data for the later processing.

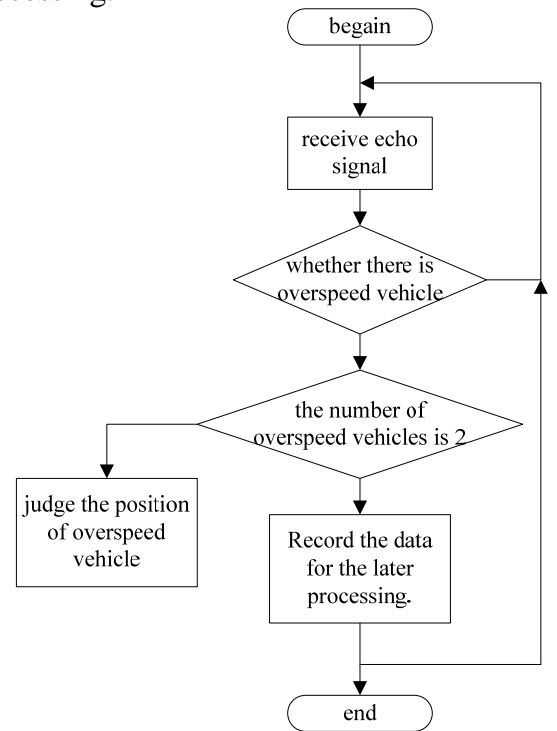

Figure 2. The diagram of overspeed vehicles measuring

\section{B. Improving the precision of multi-target measuring}

According to signal processing principle, the inherent resolution of signal determine the identification ability of system. The most important character of radar echoes is the variation of spectrum with time of each ingredient signal. Time-frequency analysis can achieve higher identification ability. The paper utilizes STFT to improve time-frequency resolution [6].

Let $s(n), 1 \leq n \leq T$ represent echo signal, $f_{s}$ represents sample frequency. The procedure of echo measuring is as the following.

Step 1: Determine the window function and window width and computer the spectrum $S T F T_{s}(n, \omega)$.

Step 2: Divide STFT spectrum into several sections in time axis to guarantee that each section is a linear FM signal. Then compute Radon transformation $\operatorname{RSTFT}_{s}(u, a)$ and $\left|\operatorname{RSTFT}_{s}(u, a)\right|$ for each section.

Step 3: Find the revolve angle and frequency position according to the maximum value of $\left|\operatorname{RSTFT}_{s}(u, a)\right|$. And compute the initiative frequency and linear FM value.

Step 4: Compensate the strongest linear FM ingredient to single frequency ingredient. Meanwhile keep other ingredients invariant.

Step 5: Design band-resistant filter to degrade the strongest ingredient.

Step 6: Obtain the initiative form of echo signal which has been filted.

Step 7: Repeat step 3 to step 6 till there is no linear FM ingredient which can be detected.

\section{SIMULATION AND EXPERIMENTS}

\section{A. Simulation}

Define abbreviations and acronyms the first time they are used in the text, even after they have been defined in the abstract. Abbreviations such as IEEE, SI, MKS, CGS, sc, dc, and rms do not have to be defined. Do not use abbreviations in the title or heads unless they are unavoidable.

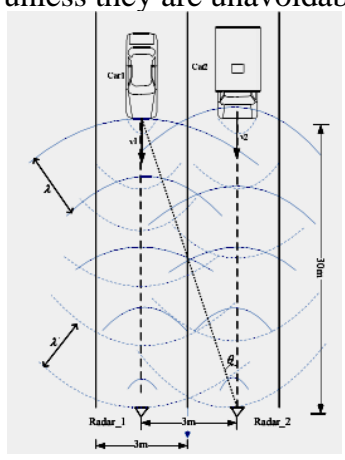

Figure 3. Speed measuring model

Use the speed measuring model shown in Figure 3 to verify the algorithm proposed in the last section. The signal model is as the (2). 


$$
X(t)=\sum_{i=1}^{m} k_{i} a_{i} \sin \left(2 \pi f_{d i} t+\varphi_{i}\right)+\rho_{\omega} \delta(i)
$$

$a_{i}$ and $f_{d i}$ represent the amplitude and frequency of echo signals respectively. ${ }^{k_{i}}$ represents amplitude gain coefficients. $\delta(i)$ represents white noise. Suppose the speed region of vehicles is $5 \mathrm{~km} / \mathrm{h} \sim 300 \mathrm{~km} / \mathrm{h}$, then Doppler frequency shift region should be $222.2 \mathrm{~Hz} \sim 13333 \mathrm{~Hz}$, choose sampling frequency $f_{s}=4 \times 13333=53 \mathrm{KHz}$, the number of sampling points is 512 .

Suppose the speed limited is below $100 \mathrm{~km} / \mathrm{h}$ and the vehicle speeds of two lanes are $v_{1}=160 \mathrm{~km} / \mathrm{h}, \quad v_{2}=80 \mathrm{~km} / \mathrm{h}$ respectively. Then we obtain simulation graphics shown in Figure 4.
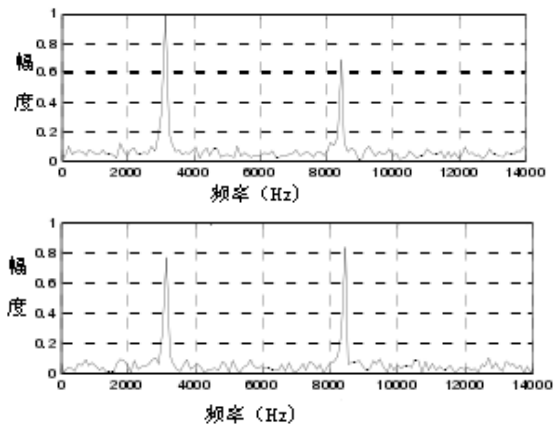

Figure 4. The simulation graphics

Utilize the algorithm provided in the last section to compute vehicle speed. According to the first sampling results, the overspeed vehicle is in left lane. According to the second sampling results, the speed is obtained. The error region is below.

\section{B. Experiments}

Figure 5 and Figure 6 are shot in a section of a highway.

Figure 5 was shot in daytime. Figure 6 was shot at night.

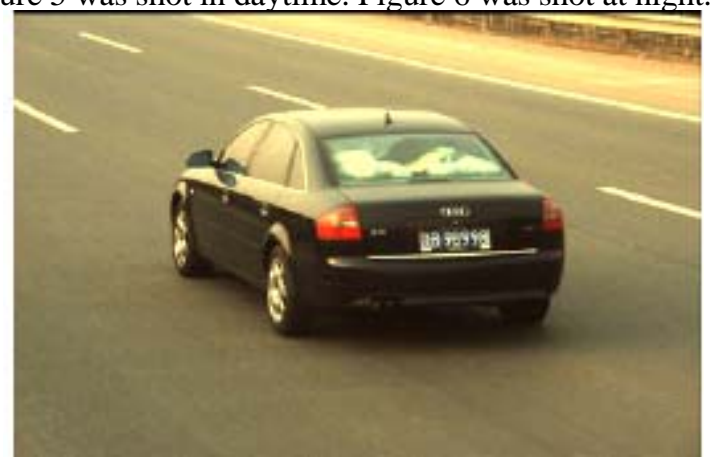

Figure 5. Single vehicle

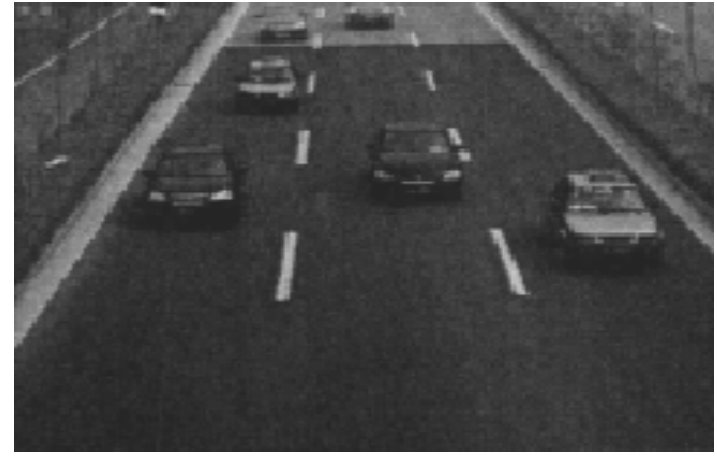

Figure 6. Several vehicles

Utilize the algorithm provided in the last section to compute vehicle speed in Figure 6. Multiple ingredients of echo are obtained and parameters are computed. According to echo corresponding to each vehicle, we obtain the speed of the front vehicles respectively in the three lanes. The speed values are $96 \mathrm{~km} / \mathrm{s} 、 122 \mathrm{~km} / \mathrm{h}$ and $109 \mathrm{~km} / \mathrm{h}$ from left to right. Meanwhile the echoes reflected from hinder vehicles are handled as noises for their weakness.

\section{CONCLUSION}

Radar's velocity measurement plays an important role in studying the target's characteristics, the target's orbit confirmation and missile's safe controlling. This paper advises to choose appropriate frequency and adopt acceleration compensation to improve speed measuring precision for single target circumstance. Furthermore it provides a method based on time-frequency analysis to identify multi objects. It also verifies the effectiveness of the algorithm for multi-target circumstance through simulation and experiments.

\section{REFERENCES}

[1] Y. Chen, "High speed pulse Doppler recognition,” Nanjing University of Science and Technology, Nanjing, 2005

[2] R.P. Li, "Research and Design on TT\&C of Launch Test Range Digital Simulation System,” National University of Defense Technology, Changsha, 2005

[3] Y.Y. Gao, F. Luo, S.J. Wu, "Study of High Speed Real-time Signal Processing System of Tracking Radar,” Modern Electronic Technique, vol. 268, pp. 19-21, 2008

[4] M. Xu, "A Study on Velocity Measurement for Monopulse instrumentation Radar,” Modern Radar, Vo.127 , No.1, pp. 58-61, January 2005

[5] B.J. Xu, "Multiple frequency CW Ranging Radar critical theory, algorithm and software and hardware design," National University of Defense Technology, Changsha, 2001

[6] Q. Xu, "Design of Signal and Research of information Extraction to high resolution Radar," University of Electronic Science and Technology of China, Xian, 2002 